Bolm. Zool., Unlv. S. Paulo

5: $149-168,1980$

\title{
FASCICULONEMERTES ARENICOLA GEN. ET SP. N. (HOPLONEMERTINI MONOSTILIFERA) DE CHILE CENTRAL
}

\author{
Malva Sánchez y Juan Cancino \\ Laboratorio de Zoologia \\ Departamento de Biologia Ambiental y \\ de Poblaciones \\ Instituto de Ciencias Biológicas \\ Universidad Catolica de Chile \\ Casilla 114-D. Santiago \\ (Recebido em 03.09.1979)
}

\section{RESUMO}

Descrita um a nova espécie de hoplonemertino monostllifero coletada numa praia arenosa em s Molles, Chile Central. Para sua identificação as seguintes características foram consideradas: a musculatura longitudinal da porção anterior do corpo é dividida em 2 camadas por tecido conjuntivo; ausência de septo precerebral; músculos retratores da cabeça relacionados à camada interna da musculatura longitudinal; existência de musculatura diagonal e dorso-ventral. Outras caracteristicas taxonômicas encontradas foram: células do neurocordão presentes no gânglio ventral; cordão nervoso lateral com um só núcleo de fibras; órgāos cerebrais localizados anteriormente ao cérebro e canal cerebral com bifurcaçāo curta; vasos sangüineos laterais atravessando o anel cerebral; bainha muscular da probóscide disposta em 2 camadas separadas; bainha da probóscide alcança o terço posterior do corpo.

Essa combinação de caracteres excluem esta espécie de qualquer Monostillfera conhectdo e por isso um gênero novo está sendo proposto.

\section{SUMMARY}

A new monostiliferous hoplonemertean collected in a sandy beach at Los Molles, Central Chile is described. For this characterization the following featu'es have been mainly considered: a longitudinal musculature of the anterior sortion of the body which is divided into two layers by a connective tissue; ab- 
sence of precerebral septum; head retractor muscles related to the inner longltudinal musculature layer; existence of a diagonal and dorsoventral musculature. Other taxonomic characteristics found are: neurochord cells present in the ventral ganglia; lateral nerve cords with one fibrous core; cerebral organs located anterior to the brain and a cerebral canal with a short bifurcation; lateral blood vessels passing through the cerebral ring; proboscis sheath musculature arranged in two separate layers; the proboscis sheath reaches the posterior third of the body.

These combination of features excludes this species from any of the known genera of Monostilifera and thus a new genus is proposed for it.

\section{INTRODUCION}

Durante el estudio de la macrofauna en una playa de arena en la Zona Central de Chile, Los Molles, se recolectó una población de nemertinos que resultaron ser hoplonemertinos monostilifera. La especie exhibe características que la diferencian claramente de los géneros ya descritos, por lo tanto se considera preciso establecer un nuevo género para ella. En el presente trabajo se describe Fasciculonemertes arenicola gen. et sp. n. y se discuten los rasgos considerados taxonómicamente importantes.

\section{MATERIALES Y METODOS}

Los especímenes se recolectaron en una playa de arena de la localidad de Los Molles en la zona intermareal, a $30 \mathrm{~m}$ de la berma hacia el mar, entre 10 y $15 \mathrm{~cm}$ de profundidad. Fauna acompañante fueron: Emerita analoga (Decapoda, Anomura), Mesodesma donacium (Mollusca, Bivalvia), un poliqueto de la Familia Onuphidae y un nemertino Lineidae.

Los ejemplares se estudiaron vivos em acuarios con agua de mar recirculada a $13^{\circ} \mathrm{C}$ y cuatro de ellos en cortes histológicos seriados, para lo cual fueron narcotizados em $\mathrm{MgCL}_{2} 6 \mathrm{H}_{2} \mathrm{O}$ en solucion al $7 \%$ en agua potable; fijados en Bouin alcohólico; incluídos en parafina; cortados transversales de $7 \mu \mathrm{m}$ y teñidos con Hematoxilina Fluoxina, algunos de estos cortes se trataron con la reacción de P.A.S. Se hicieron además secciones longitudinales de la región anterior teñidas con Van Gieson.

\section{DIAGNOSIS}

Fasciculonemertes gen. n. Hoplonemertini, Monostilifera con cuerpo cilíndrico y alargado. Organos cerebrales situados anteriores a los ganglios cerebroides, canales cerebrales con bifurcación corta. Ganglios cerebroides con neurilema y células neurocordales. Cordones nerviosos laterales con un núcleo de fibras. Comisura nerviosa anal supraintestinal. El esófago se abre en el rincodeo. Porción anterior del intestino con divertículos no ramificados, porción posterior con divertículos ramificados. Los vasos laterales pasan a través del anillo cerebral; el vaso dorsal se origina a partir del lateral izquierdo, ubicándose en la primera parte de su recorrido dentro de la vaina de la proboscis. Pared del cuerpo con musculatura diagonal y dorsoventral. En la región anterior del cuerpo la musculatura longitudinal está dividida en dos capas por tejido conectivo, la porción interior forma la inserción de la proboscis $y$ 
los retractores de la cabeza. No hay septo precerebral. Musculatura de la vaina de la proboscis formada por dos capas separadas; rincoceloma alcanza hasta el tercio posterior del cuerpo.

\section{DESCRIPCION}

Fasciculonemertes arenicola gen. et. sp n.

Holotipo, 1 especímen hembra en cortes histológicos seriados, depositado en el Museo Nacional de Historia Natural de Santiago, Chile (NT 70004), col. M. Sánchez y J. Cancino. Lccalidad tipo, Los Molles, Chile Central $\left(32^{\circ} 15^{\prime}\right.$ Lat. S., $71^{\circ} 33^{\prime}$ Long. W.). Paratipos, 1 especímen en cortes seriados, 1 especímen in toto depositados en la Sala de Sistemática, Laboratorio de Zoologia, Universidad Católica de Chile. Provienen de la localidad tipo.

Etimología, Fasciculonemertes se refiere a la musculatura longitudinal dispuesta en fascículos y arenicola al habitat de la especie.

\section{Morfología externa y conducta.}

Los especímenes sexualmente maduros midieron aproximadamente $120 \mathrm{~mm}$ de largo y $4 \mathrm{~mm}$ de díametro. El lóbulo cefálico es corto, aguzado y está levemente separado del cuerpo por un par de surcos cefá-

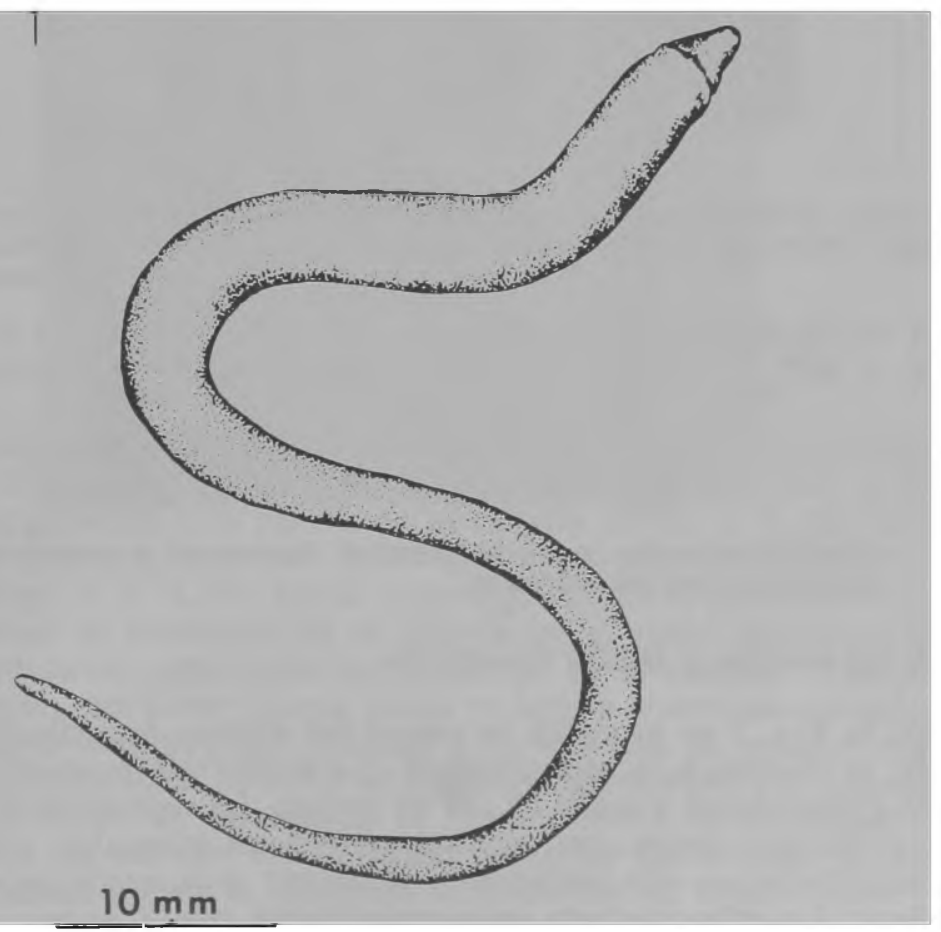

Fig. 1 - Fasciculonemertes orenicola. Animal completo, vista dorsal. 
licos poco profundos ubicados detrás de la abertura del rincodeo, el dorsal es incurvado hacia adelante, el ventral recto. A cada lado y anterior a la abertura del rincodeo hay 2 ocelos pequeños y oscuros. El cuerpo es cilíndrico, más delgado de la mitad hacia atrás (Fig. 1); con los dos tercios anteriores de color rojo anaranjado y el tercio posterior blanquecino, estos colores se pierden con la fijación.

Los gusanos son muy contráctiles y al estimularlos evierten violentamente la proboscis que es cilíndrica y del mismo color del cuerpo. Mantenidos en acuarios con arena se entierran en el sustrato formando tubos mucosos con agregaciones de arena, después de tres días en estas condiciones comienzan a fragmentarse por el extremo posterior.

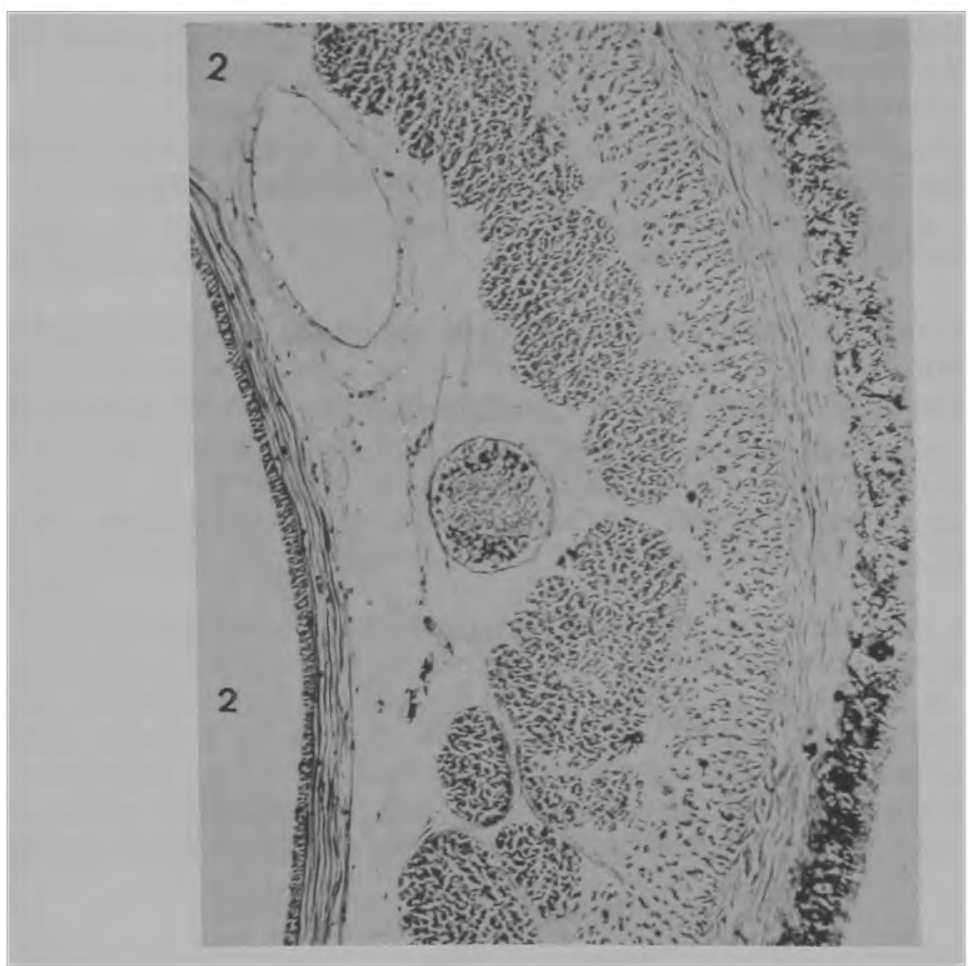

Fig. 2 - Fasciculonemertes arenicola. Sección transversal a través de la pared del cuerpo a nivel del estómago.

Pared del cuerpo y tejido conectivo.

En la Fig. 2 se muestra la pared del cuerpo constituída por la epidermis, la dermis y la musculatura. La epidermis presenta abundantes células glandulares y mide $20 \mu \mathrm{m}$ de grosor. La dermis está formada por tejido conectivo denso con escasas células, es irregular y forma pequeñas evaginaciones que parecieran penetrar a la epidermis, mide $5 \mu \mathrm{m}$ de grosor. La musculatura está constituída por 3 capas: una circular externa que llega a la punta de la cabeza cuyo grosor varía entre 15 y $30 \mu \mathrm{m}$, una diagonal intermedia que se encuentra en la mitad anterior 


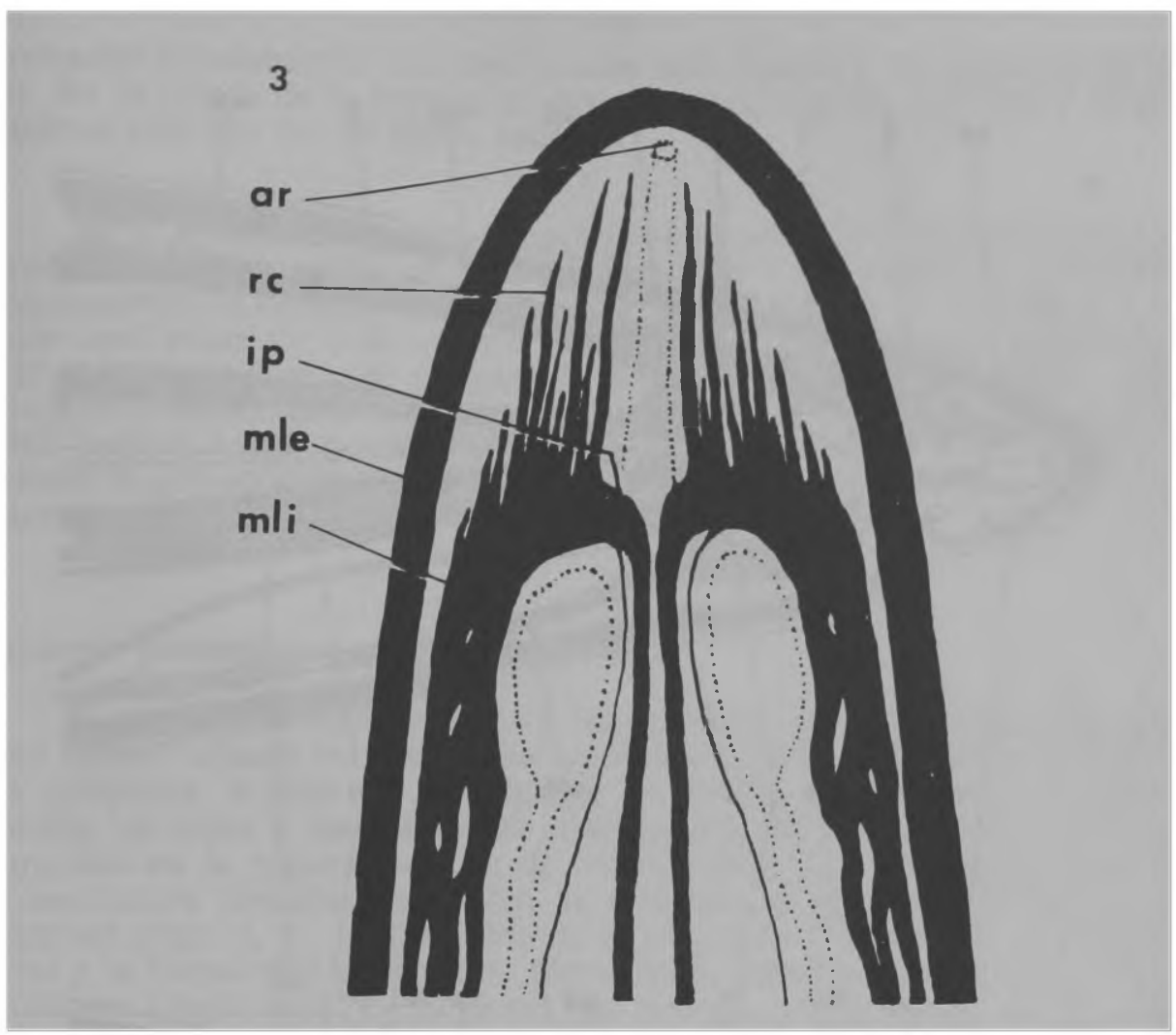

Fig. 3 - Fasciculonemertes arenicola. Representación de la musculatura longitudinal de la pared del cuerpo y sus derivados precerebrales, siguiendo esquema de Kirsteuer (1974).

del cuerpo y mide de 7 a $11 \mu \mathrm{m}$ de grosor; una longitudinal interna comparativamente más gruesa que las dos anteriores (100 a $360 \mu \mathrm{m}$ de grosor).

Del píloro hacia adelante la musculatura longitudinal interna está dividida en 2 capas por tejido conectivo: una exterior laxa y delgada (Fig. 2), que alcanza a la punta de la cabeza (Figs. 3, 4, 5), otra interior más compacta que forma fascículos separados entre sí por tejido conectivo (Figs. 2, 3, 4,5). En la régión precerebral parte de esta musculatura forma la inserción de la proboscis, el resto continua fasciculada hacia adelante como músculo retractor de la cabeza (Figs. $3,4,5$ ). Desde la región del píloro hacia atrás el tejido conectivo situado entre la musculatura longitudinal exterior y la interior se reduce, los fascículos de la longitudinal interior se hacen progresivamente menos conspicuos hasta perderse, quedando la musculatura longitudinal constituída sólo por un estrato. En la mitad posterior del cuerpo la musculatura longitudinal presenta interdigitaciones con el tejido conectivo, no alcanzando las proyecciones de este último a la capa circular (Fig. 6). También se encontró en esta porción del cuerpo, fibras dorso-ventrales abundantes dispuestas entre la musculatura longitudinal (Fig. 7), y 


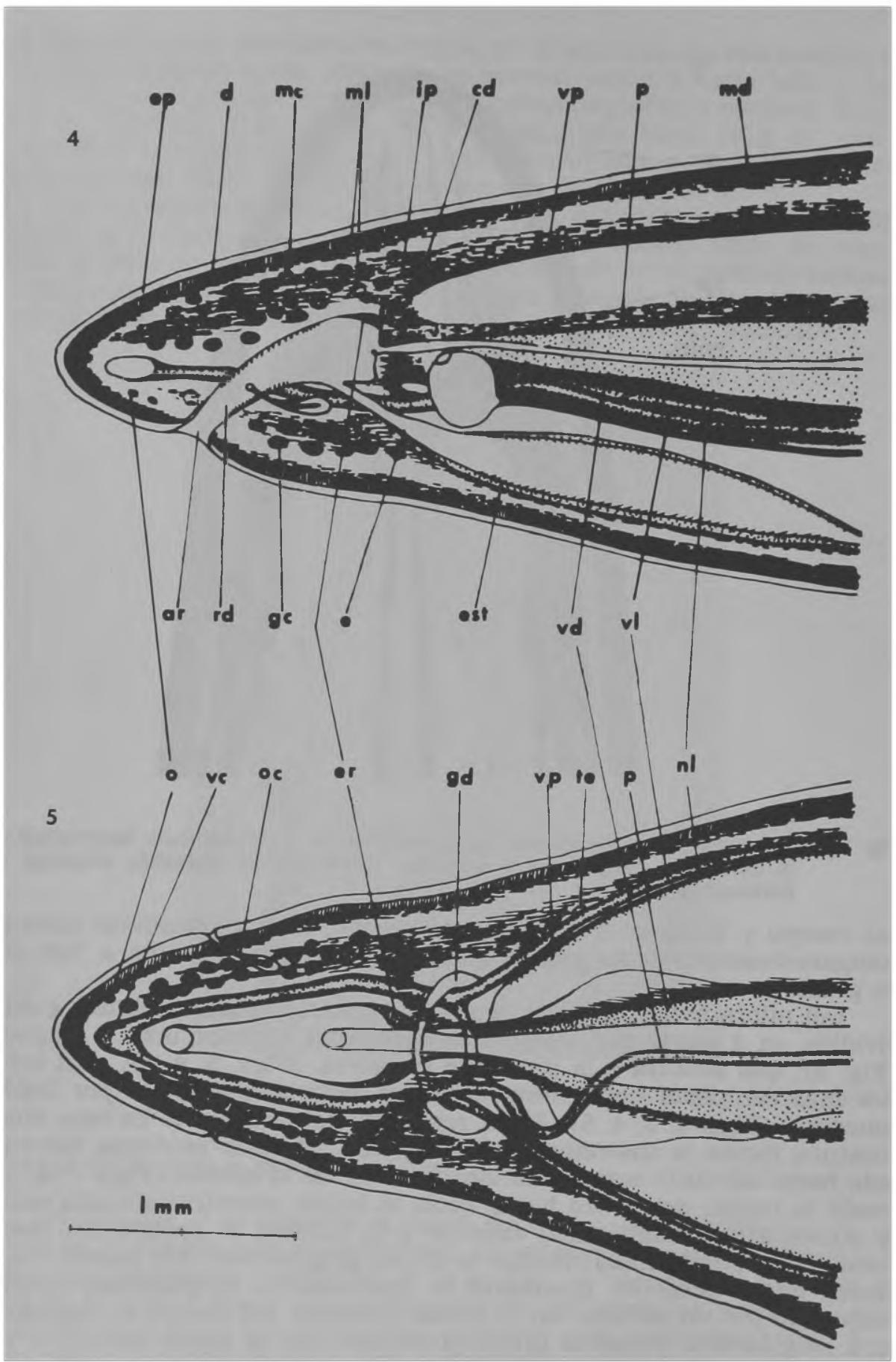

Figs. 4-5 - Fasciculonemertes arenicola. Reconstrucción de la porción anterior del cuerpo a partir de cortes transversales; (4) en vista lateral, (5) en vista dorsal. 
rodeando parcialmente los divertículos intestinales y las gónadas (Fig. 8). En la punta de la cabeza además de musculatura circular y long1tudinal hay una red de fibras radiales.

En F. arenicola el tejido conectivo es abundante y concordando con Pedersen (1968), está constituído por fibras de colágeno, sustancia fundamental y células. Con la tinción de Van Gieson se observó que en este caso posee un componente filamentoso laxo, sustancia fundamental abundante y células escasas. Se distribuye entre las glándulas cefálicas y la musculatura longitudinal anterior, asimismo las fibras pueden formar haces que circundan los vasos sanguíneos, cordones nerviosos y estructuras tubulares. Sólo el tejido conectivo que forma la dermis es denso.

\section{Aparato proboscídeo}

El rincodeo se abre ventral y subterminal (Fig. 4). Está revestido por epitelio ciliado con glándulas abundantes que se tiñen como las de la epidermis. A nivel de los órganos cerebrales el epitelio se adelgaza, pierde los cilios y las glándulas disminuyen. El esófago se abre en el rincodeo en la región anterior al cerebro (Fig. 4). Trás esta unión la musculatura circular que rodea al rincodeo se engruesa y forma un esfinter (Figs. 4, 5). La inserción de la proboscis (Figs. 3, 4), es precerebral y se forma sólo a partir de fibras de la porción interior de la musculatura longitudinal de la pared del cuerpo, por lo tanto, de acuerdo con Friedrich (1939) y Kirsteuer (1974), consideramos que no hay septo precerebral.

Detrás de la inserción, la vaina de la trompa está formada por un endotelio que la reviste y por una túnica muscular con fibras circulares. Posteriormente entre la capa circular y el endotelio aparece una capa longitudinal (Figs. 2, 9), esta disposíción se conserva hasta el término de la vaina en el tercio posterior del especímen. El grosor de la musculatura varía, aunque es evidente un engrosamiento paulatino a medida que se acerca al final, un corto trecho antes de terminar la vaina disminuye gradualmente de díametro hasta desaparecer por debajo de la región dorsal de la pared del cuerpo. No hay divertículos rincocelómicos.

La trompa está constituída por una cámara anterior, un diafragma, una vesícula bulbosa y una cámara posterior.

1. - La cámara anterior presenta: un epitelio de tipo glándular mucosecretor, que forma pliegues longitudinales de altura y largo variable soportados por extensiones de la capa conectiva subyacente; una túnica muscular formada por una capa circular externa, algo laxa en 

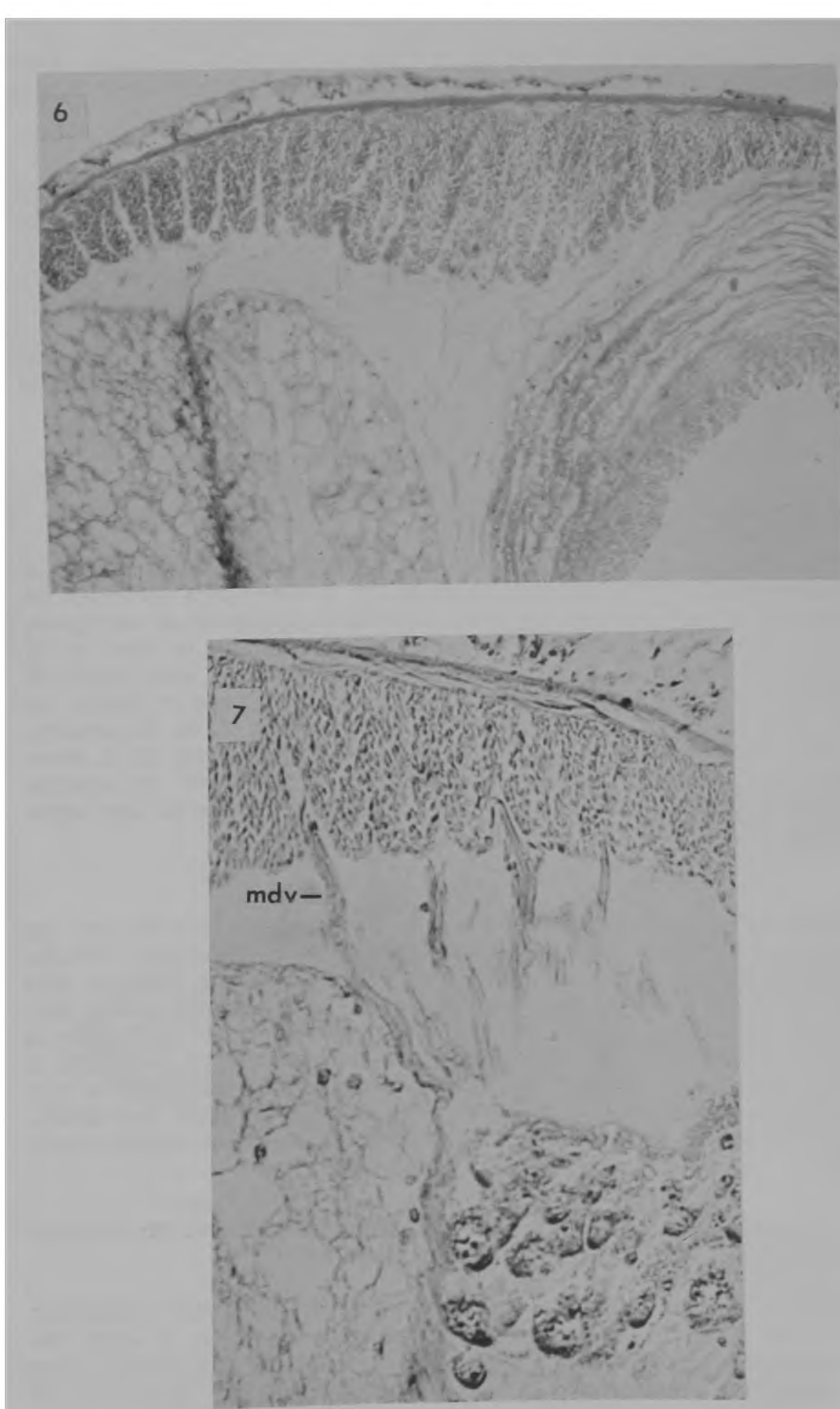


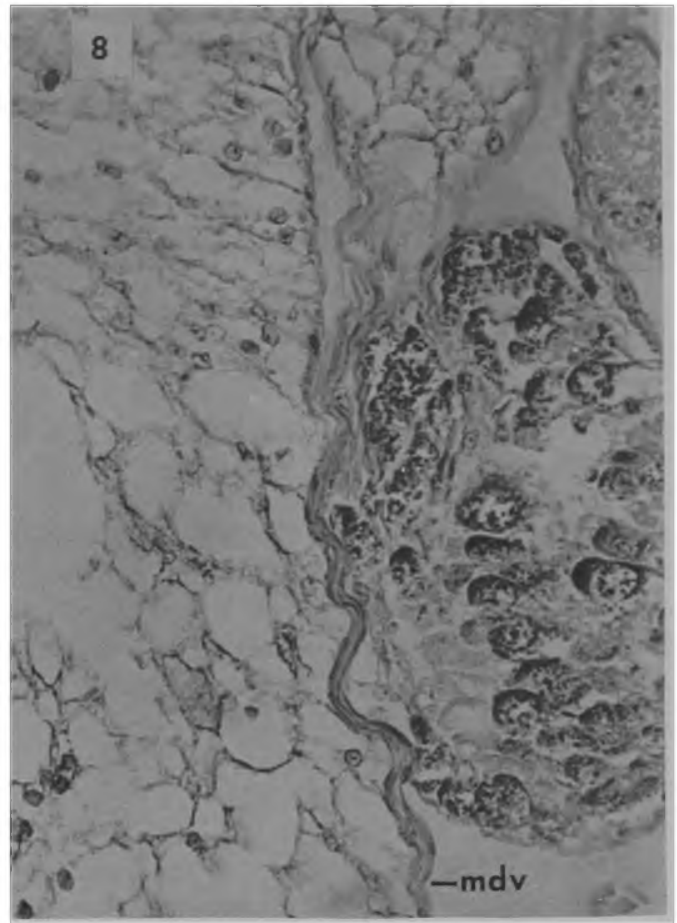

Figs. 6-8 - Fasciculonemertes arenicola. Cortes transversales a nivel del intestino mostrando: (6) interdigitaciones de la musculatura longitudinal de la pared del cuerpo con el tejido conectivo; (7-8) musculatura dorso ventral de la pared del cuerpo.

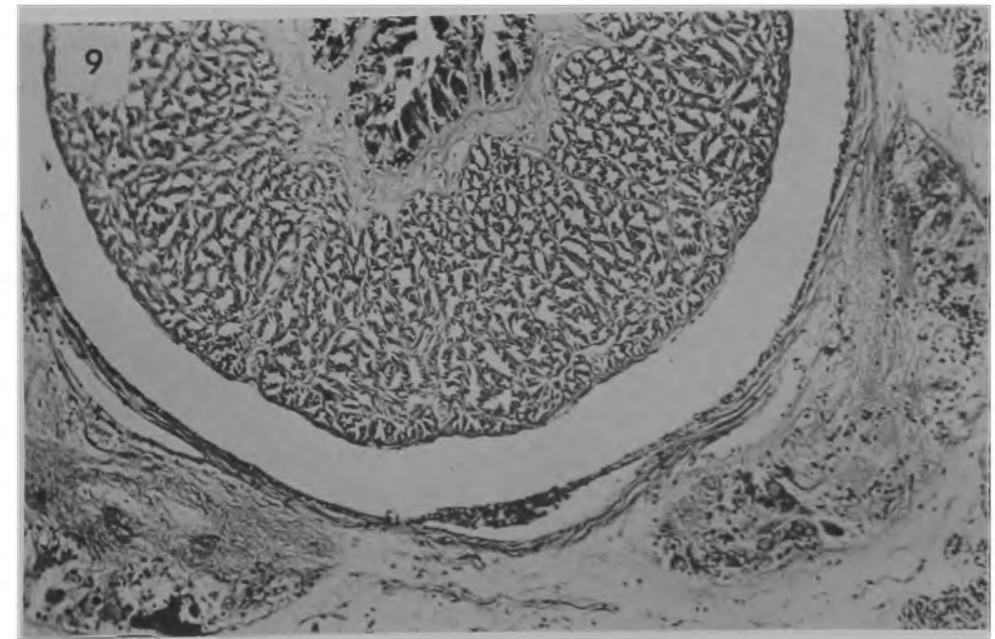

Fig. 9 - Fasciculonemertes arenicola. Corte transversal por la región cerebral; vasos laterales dentro del anillo cerebral, vaso medio dorsal en la pared de la vaina de la proboscis. 
la región anterior, y una capa longitudinal interna (Fig. 9). La proboscis en toda su extensión está revestida internamente por un epitelio plano simple y continuo.

Los nervios de la trompa son 10 y se distinguen sólo en la cámara anterior, dispuestos longitudinalmente y conectados a través de un plexo circular, el cual separa la musculatura longitudinal interna en dos; siendo la porción externa la más gruesa y equivalente a las 3/4 partes del espesor total del estrato.

2. - El diafragma contiene la armadura de la trompa, que consiste en un estilete principal alargado de superficie lisa, una base cilíndrica (Fig. 10) y un número variable de bolsas de estiletes accesorios (para medidas ver Tabla I). En el tejido conectivo ubicado entre estas rolsas se localizan numerosas glándulas acidófilas.

3 . - La vesícula es bulbosa, con gruesas paredes musculares en las cuales se distinguen claramente 2 capas, una longitudinal externa y otra más gruesa circular e interna. Además se observan algunas fibras diagonales, dispersas entre las circulares.

4. - La cámara posterior comparativamente es de díametro pequeño, con paredes delgadas y escaso tejido conectivo entre el epitelio y la musculatura. El epitelio está formado por células glandulares con gruesas granulaciones citoplasmáticas agrupadas en formaciones papiliformes de base angosta. La túnica muscular posee una capa longitudinal externa cuyas fibras se proyectan y sostienen a las prolongaciones papiliformes, y una circular interna de poco espesor que en algunos lugares es de una sola fibra.

Esta porción de la trompa está conectada a la cámara anterior a través de un conducto que atraviesa el diafragma y la vesícula bulbosa. A nivel del diafragma el conducto es muy fino y está revestido por

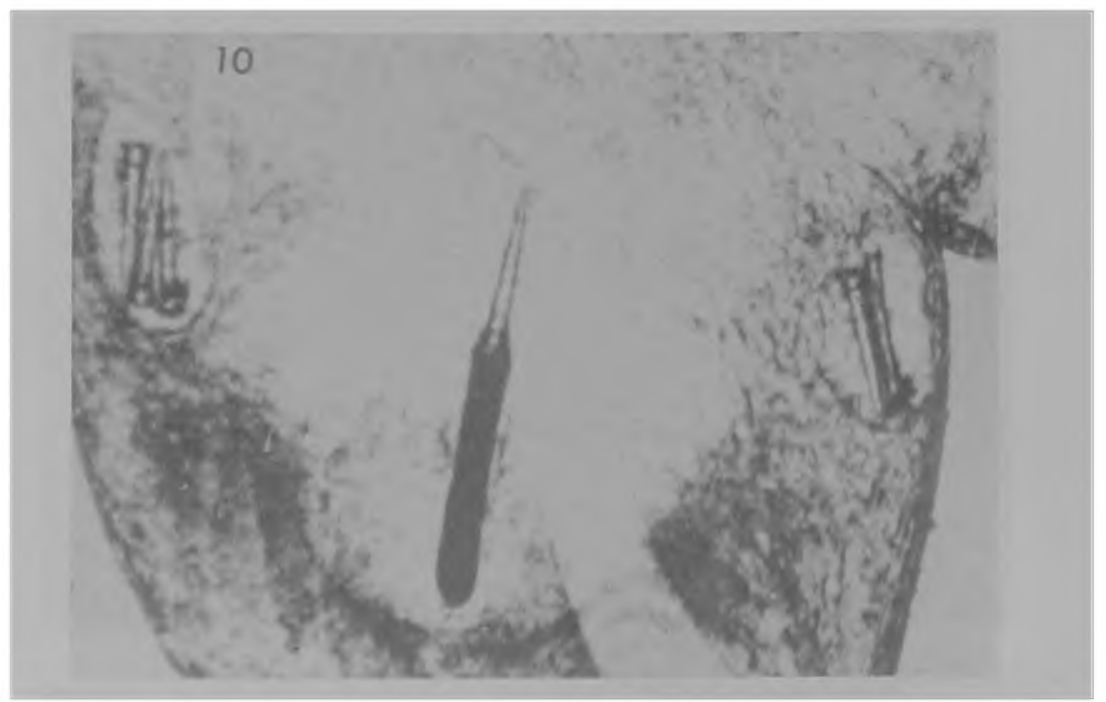

Fig. 10 - Fasciculonemertes arenicola. Armadura de la probosis comprimida entre porta y cubre. 
un epitelio plano simple, al desembocar en la vesícula se ensancha presentando un epitelio seudoestratificado, con células de citoplasma apical basófilo.

A medida que se acerca el término de la trompa, las agrupaciones papiliformes de la cámara posterior se van haciendo cada vez más cortas hasta desaparecer; se considera que en este punto nace el músculo retractor de la trompa, que es la continuación de las fibras longitudinales de la cámara posterior, las cuales como un fascículo compacto, recorren un corto espacio para luego insertarse en la región dorsal de la vaina; a este nivel las fibras longitudinales del retractor se continuan con las fibras de la capa longitudinal interna de la vaina.

\section{Sistema nervioso.}

Los ganglios cerebroides se ubican en la zona de transición entre esófago y estómago (Fig. 4), envueltos por neurilema. Los lóbulos dorsales se unen a nivel de la inserción de la proboscis por una comisura dorsal larga y delgada, los lóbulos ventrales son comparativamente más grandes y poseen una comisura corta y gruesa situada posterior a la inserción.

En la corteza ganglionar se distinguen diferentes tipos celulares, siendo los más comunes los descritos a continuación. 1. - Células pequeñas con citoplasma acidófilo de forma estrellada, núcleos esféricos intensamente teñidos con la cromatina dispuesta homogeneamente; son abundantes en el ganglio dorsal, escasas en el ventral. 2. - Células más grandes que las de tipo 1, con citoplasma teñido debilmente y de contornos definidos, núcleos esféricos u ovalados con cromatina granular distribuída en forma homogénea o periférica, nucleolo visible sólo en algunos casos; estan principalmente en el ganglio dorsal. 3. Células de forma variable con bordes definidos, el citoplasma es una fina trama rosada debilmente teñida, el núcleo es esférico y periférico con cromatina granular de densidad variable, en algunos casos se observa un nucleolo central; se ubican en el ganglio ventral. 4. - Corresponden a las células de mayor tamaño observadas, son piriformes 0 redondeadas de bordes definidos (Fig. 11). El pericarion mide en las más pequeñas $15 \mu \mathrm{m}$ de eje mayor y $6 \mu \mathrm{m}$ de eje menor; en las más grandes $42 \mu \mathrm{m}$ de eje mayor, $37 \mu \mathrm{m}$ de eje menor y $15 \mu \mathrm{m}$ el núcleo, el citoplasma es basófilo y a veces vesiculoso, el núcleo es grande y puede ser excéntrico con cromatina poco condensada, el nucleolo también es grande y excéntrico, en algunos casos hay varios nucleolos más pequeños. Hay varias en la corteza del ganglio ventral y se consideran células neurocordales.

Los cordones nerviosos laterales están formados por sólo un núcleo de fibras, emergen de los lóbulos ventrales hacia posterior (Fig. 4) y se unen a nivel del ano por una comisura supraintestinal gruesa. No hay nervio dorsal.

\section{Glándulas cefálicas y órganos de los sentidos.}

Se encontraron grandes masas de células globosas entre la musculatura longitudinal de la región precerebral, teñidas con Fluoxina y al- 


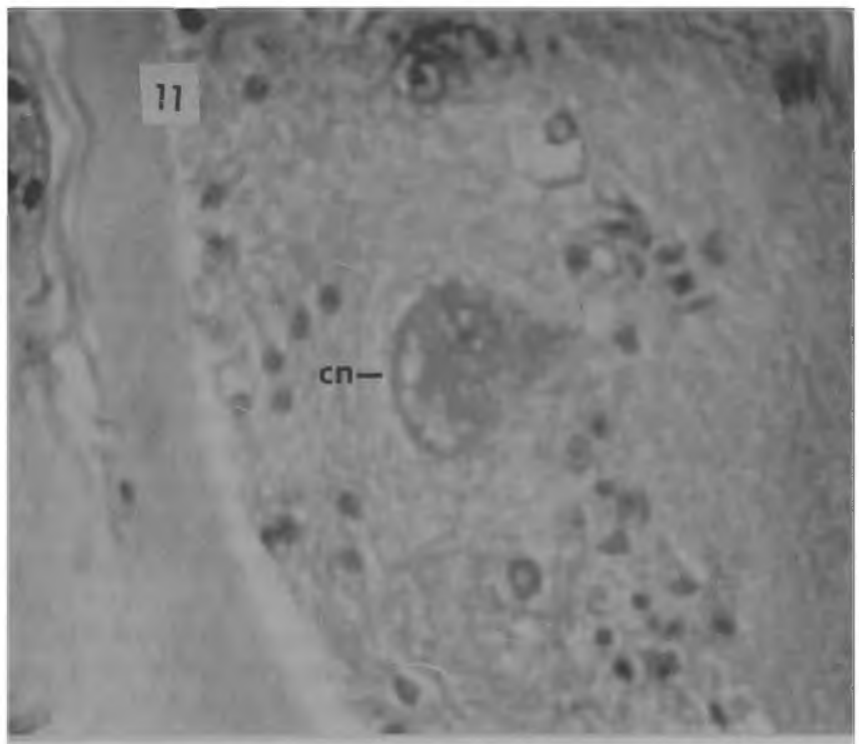

Fig. 11 - Fasciculonemertes arenicola. Corte transversal do ganglio ventral com célula neurocordal.

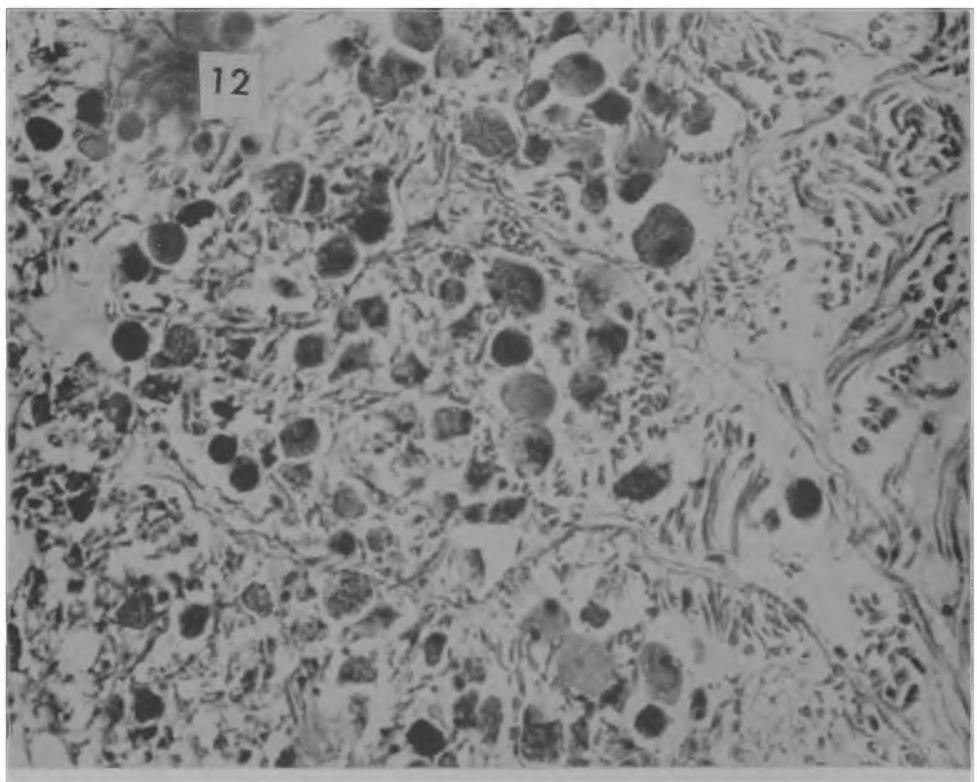

Fig. 12 - Fasciculunemertes arenicola. Corte transvereal de masas de células glandulares globosas en la región precerebral. 

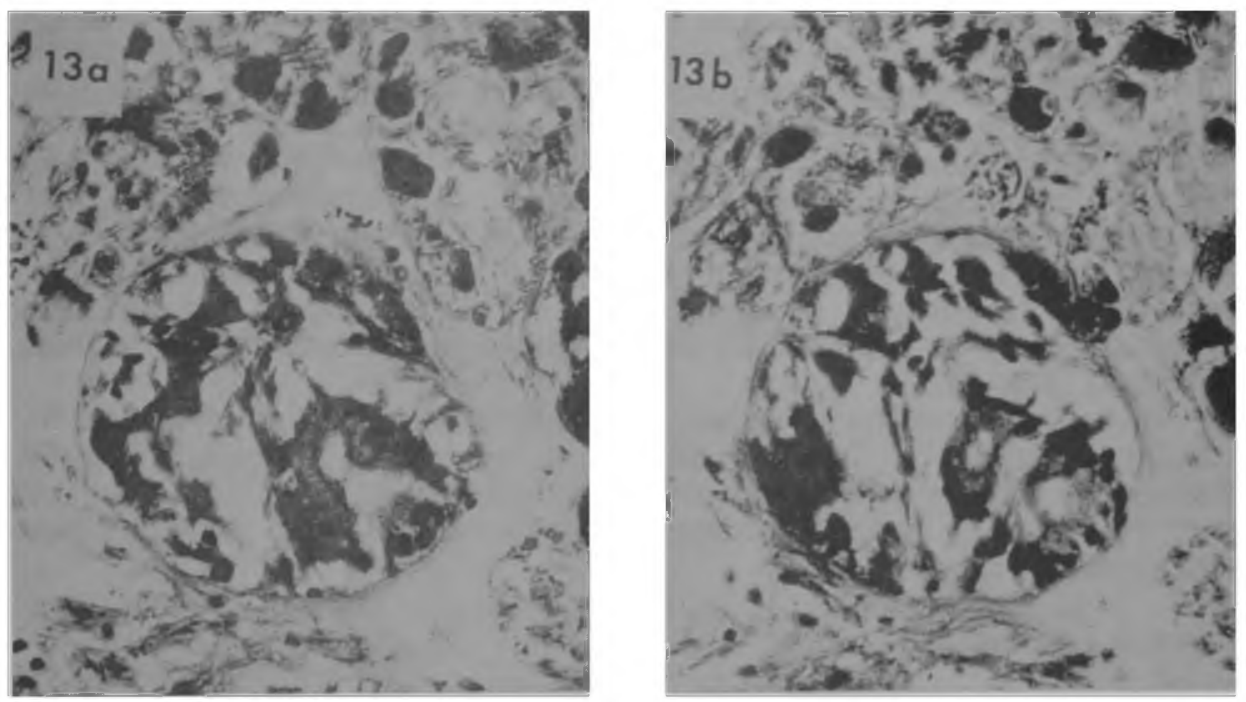

Figs. 13 - Fasciculonemertes arenicola. Cortes transversales de: (13a) canal cerepral dividiéndose; (13b) canal cerebral dividido.

gunas con Hematoxilina, por su estructura y ubicación corresponderían a glándulas cefálicas (Figs. 4, 5, 12). No es posible determinar en este momento si se trata de dos tipos celulares o de estados funcionales diferentes de un mesmo tipo. Los cortes tratados con P.A.S. dieron una reacción positiva lo que sugiere que podrían ser glicoproteínas o glicógeno, pero tratándose de glándulas cabe la posibilidad que sea una glicoproteína. La secreción sería movilizada por finos conductos excretores que no han sido claramente identificados.

Los ocelos se ubican anterior al poro de la proboscis y lateroventral al vaso cefálico (Figs. 4,5 ).

Los órganos cerebrales miden alrededor de $400 \mu \mathrm{m}$ de largo y terminan $800 \mu \mathrm{m}$ antes del cerebro (Figs. 4, 5), estan envueltos por tejido conectivo y después de penetrar la pared del cuerpo se sitúan internos a la musculatura longitudinal, estan formados por agrupaciones de células nerviosas y glandulares. El canal cerebral está revestido por células epiteliales, se abre lateralmente en el surco cefálico, recorre alrededor de $250 \mu \mathrm{m}$ y se bifurca por $28 \mu \mathrm{m}$ (Figs. 13a, 13b), para reunirse nuevamente las dos ramas en un canal.

\section{Sistema digestivo}

El esófago es un tubo estrecho que se abre en el rincodeo (Figs. $4_{\text {; }}$ 14), presenta un epitelio bajo, sin cilios con pocas glándulas, la transición al estómago es gradual y ocurre trás la inserción de la proboscis. El epitelio del estómago es alto, ciliado y provisto de abundantes glándulas acidófilas. En los 4 especímenes en que se estudió la morfología interna, al comenzar el estómago se presenta un pliegue dorsal profundo 
que se extiende por alrededor de 650 um (Figs. 14, 15), sólo en 2 casos se observa que el pliegue forma sacos cerrados internos. El epitelio del pí. loro es ciliado con pocas glándulas acidófilas, desemboca en el intestino por dorsal (Fig. 14) El ciego intestinal se proyecta hacia adclante por debajo del píloro, no muestra divertículos o bolsas intestinales (Fig. 14) ; su largo varía en diferentes animales entre $1,5 \mathrm{~mm}$ y $4,2 \mathrm{~mm}$, probablemente debido al diferente estado de contracción. El intestino en su porción anterior tiene divertículos laterales no ramificados, el intestino posterior es ramificado. El ano es ventral y subterminal. La musculatura dorso-ventral de la pared del cuerpo envuelve parcialmente al sistema digestivo (Fig. 8).

Aunque se analizaron pocos especímenes, F. arenicola presenta variación individual en la formación de sacos cerrados internos al estómago y en el largo del ciego intestinal, por lo tanto coincidimos con Berg (1972) en el sentido de no usar la parte anterior del sistama digestivo como caracter específico.

\section{Sistema circulatorio}

Los vasos están formados por un endotelio, fibras de musculatura circular y tejido conectivo. A nivel del poro de la proboscis el vaso cefálico se divide en dos vasos laterales que pasan a través del anillo cerebral (Figs. 5, 9). En la región anterior del cerebro el vaso izquierdo origina al medio dorsal, que se sitúa dentro de la pared de la vaina de la proboscis por un trecho de aproximadamente $80 \mu \mathrm{m}$ (Fig. 9). Existe entre los tres vasos anastomosis cerebral. Detrás del cerebro los vasos laterales estan dorsales a los cordones nerviosos (Fig. 2), posteiior al estómago se situán ventral a ellos. Los vasos laterales y el dorsal se unen en una anastomosis supraintestinal cerca del ano.

\section{Organos excretores.}

Se encontraron algunos túbulos excretores en posición externo lateral a los vasos sanguíneos (Fig. 5), no se detectaron conductos eferentes.

\section{Gónadas.}

Los especímenes recolectados sexualmente maduros correspondieron a hembras. Los ovarios comienzan en la mitad anterior del cuerpo y se extienden hasta el extremo posterior, ubicándose entre los divertículos intestinales en una fila a cada lado del plano medio sagital (Fig. 16). Cada gónada está constituída por un saco ventral y un gonoducto que penetra la pared del cuerpo. La dermis al ser atravesada por el ductus se hace más laxa y proyecta sus fibras hacia el epitelio (Fig. 17). Los gonoductos desembocan latero-dorsalmente sobre el cordón nervioso.

El especímen holotipo colectado em Marzo (final del verano) presentó en cada ovario de 1 a 6 huevos (Fig. 16); otro ejemplar capturado en Noviembre (comienzos del verano) alrededor de 30 huevos por saco; no se encontraron gónadas en gusanos colectados en Julio (invierno). 


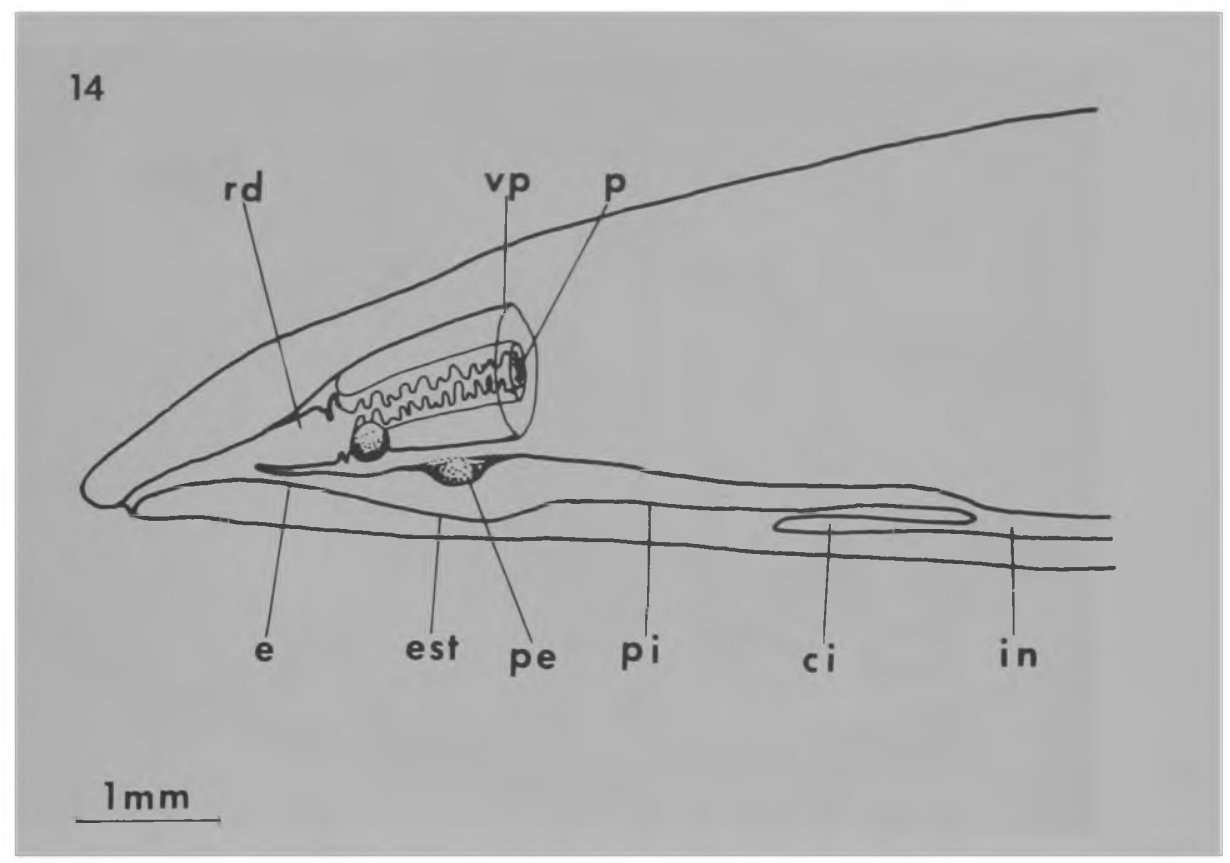

Fig. 14 - Fasciculonemertes arenicola. Reconstrucción de la región anterior del sistema digestivo, a partir de secciones transversales.

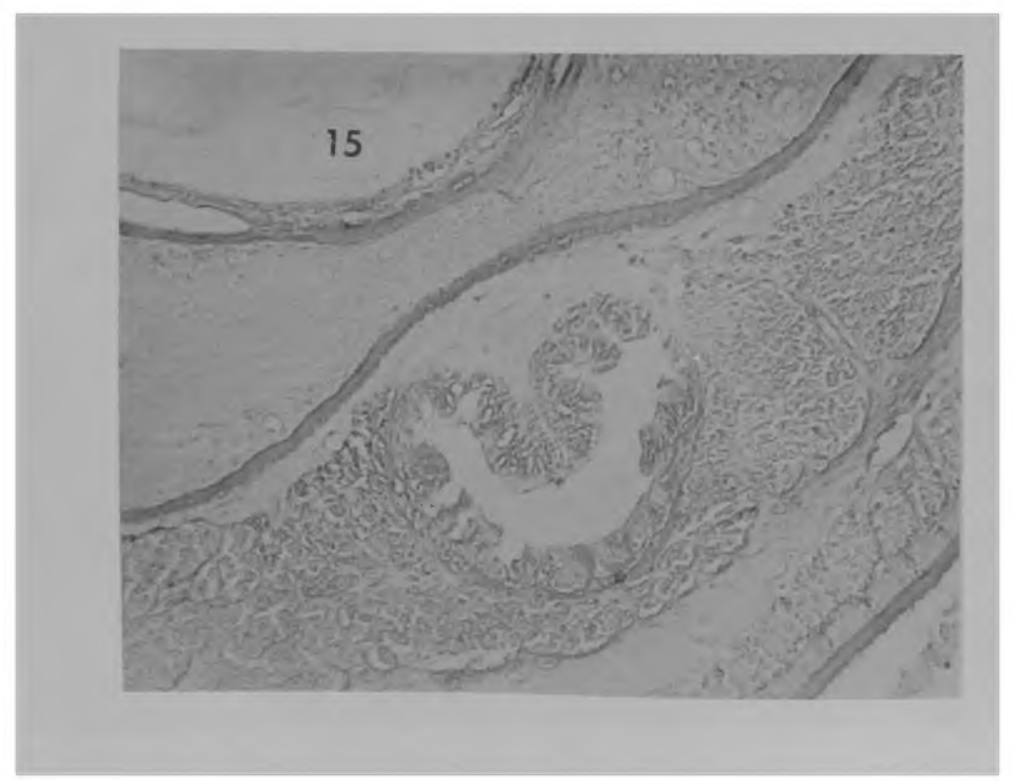
Fig. 15 - Fasciculonemertes arenicola. Corte transversal por el pliegue dorsal del es-
tómago. 

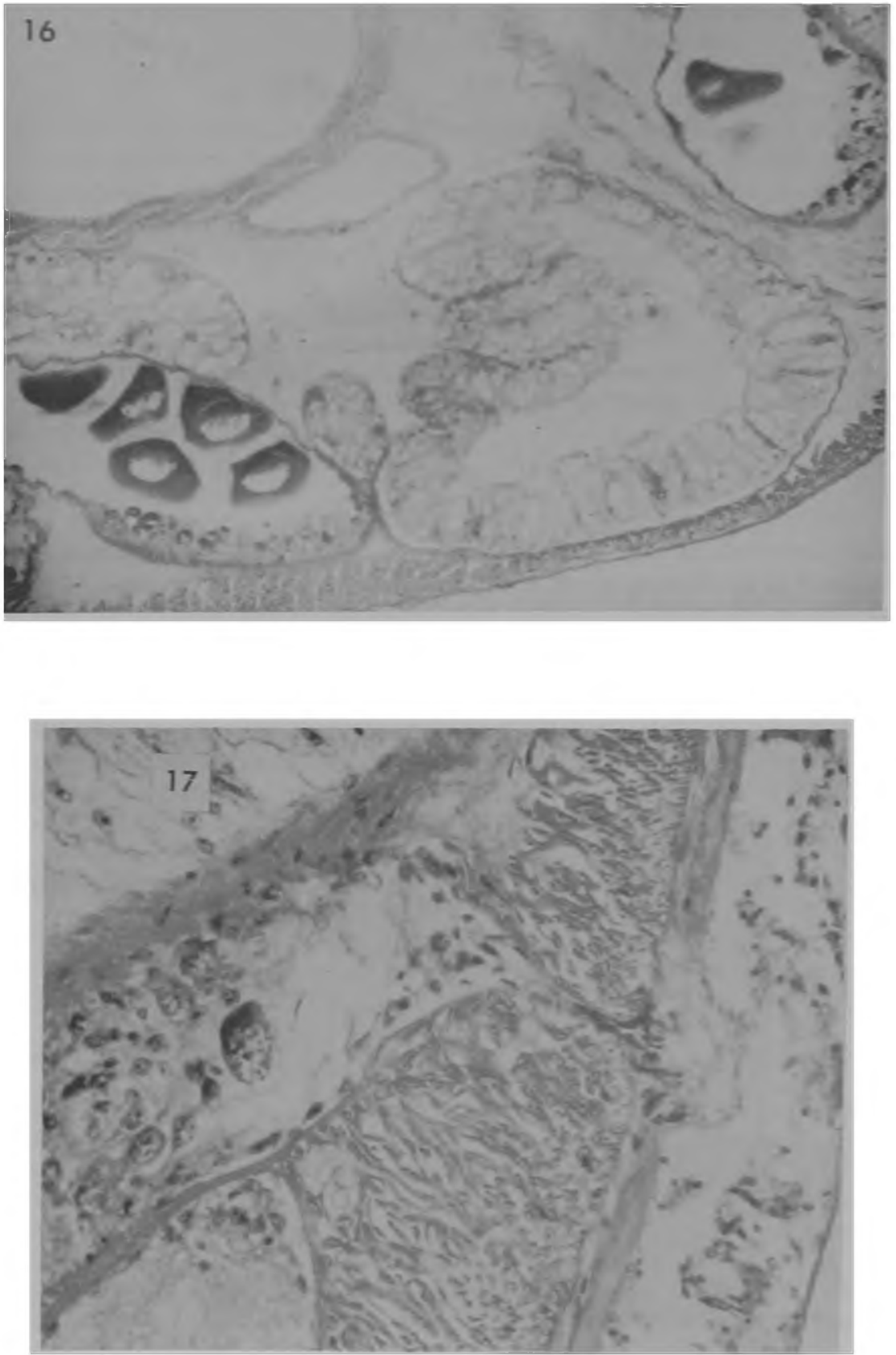

Figs. 16-17 - Fasciculonemertes arenicola. Corte transversal de un especimen hembra a nivel de las gónadas: (16) ovarios a cada lado del plano medio sagital; (17) desembocadura del oviducto a través de la pared del cuerpo. 


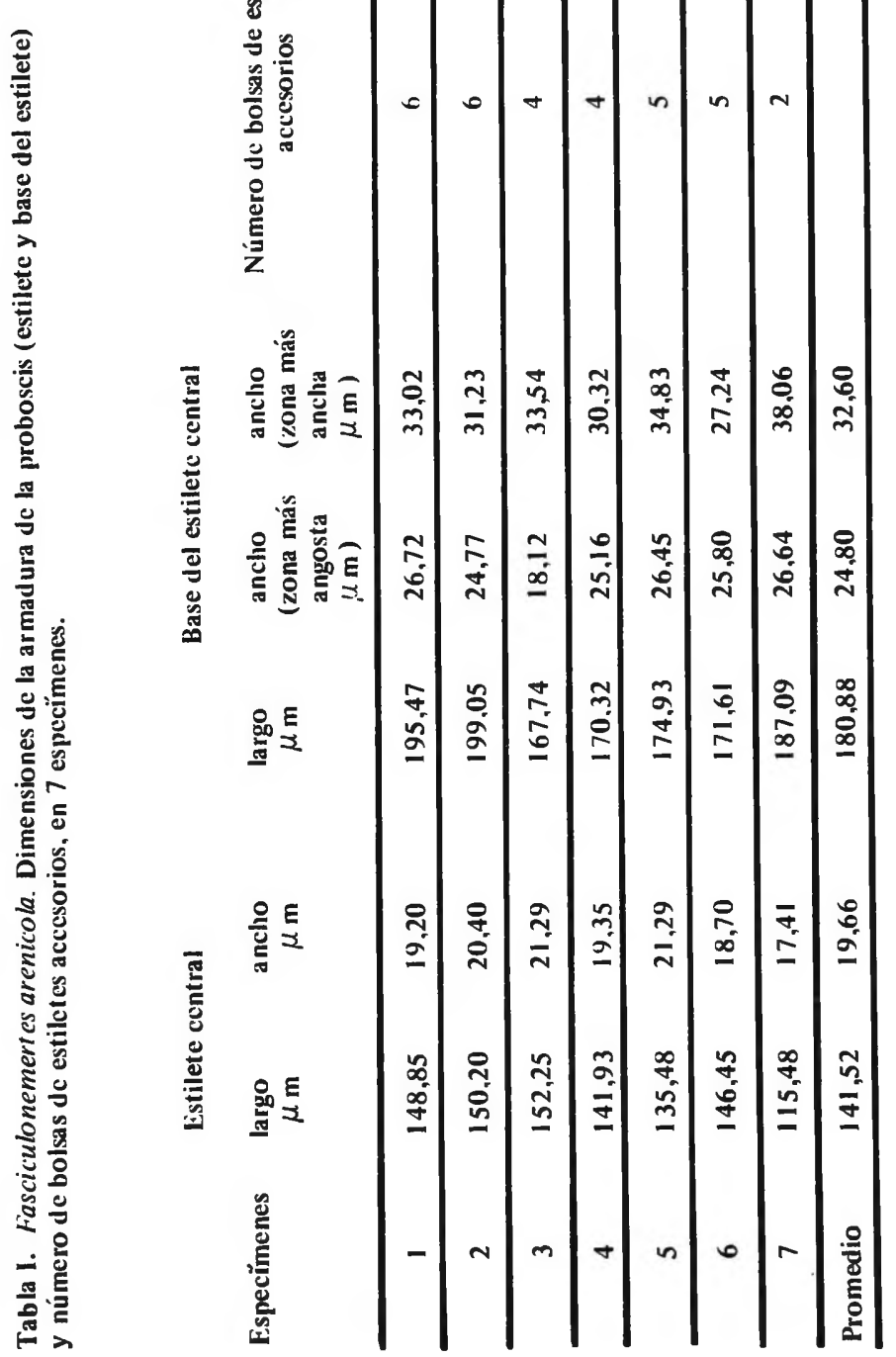




\section{DISCUSION}

Fasciculonemertes arenicola presenta un importante caracter taxonómico que es la musculatura longitudinal de la porción anterior del cuerpo dividida en dos capas. Coe $(1904,1905,1940)$ lo dá para la especie Paranemertes californica, sin embargo, Coe (1901) no lo menciona cuando erige el género Paranemertes y coloca en él las especies $\mathbf{P}$. peregrina, P. pallida y P. carnea. Coe (1944) describe Paranemertes bioccllatus y dice que: "In this species as in the others of the genus, the longitudinal musculature in the anterior portion of the body is divided into two distinct layers as described and figured by Coe $(1904,1905)$ for P. californica" Posteriormente se agregan al género especies con el caracter en cuestión como Paranemertes brattstroemi y Paranemertes sp. Friedrich (1970) y otras para las cuales no se describe como P. incola Iwata, 1952 y P. plana Iwata, 1957. Aún más, hay variación intraespecífica respecto a este rasgo morfológico en ejemplares de una misma especie colectados en diferentes localidades i. e. P. peregrina en Alaska, Coe (1901), Yamaoka (1940) en Japón, Corrêa (1964) en California.

Friedrich (1939) estabelece la especie Amphiporus korschelti y senala la importancia taxonómica que reviste la presencia de musculatura longitudinal dividida en la región anterior y la relación de ella con la inserción de la trompa; Friedrich (1955) separa del género Amphiporus a un grupo de especies cuyas relaciones no son bien claras, denominándolo grupo Amphiporus hastatus, considerando para ello entre otros elementos una doble capa de musculatura longitudinal, asimismo asume que Paranemertes y Nemertes Johnston, 1837 son Monostilifera que exhiben este carácter. Otıos géneros reportados con esta particularidad miológica son: Paramphiporus Kirsteuer, 1965; Poseidonemertes Kirsteuer, 1967; y Correanemertes Kirsteuer, 1967. En base principalmente a disposición de la musculatura longitudinal en dos capas, composición del septo precerebral e inserción de la proboscis, y orígen de los retractores de la cabeza, Kirsteuer (1974) revisa los géneros y especies relacionados con estos caracteres, insistiendo en el valor taxonómico que ellos tienen como elementos de diagnosis a nivel genérico.

Al comparar Fasciculonemertes arenicola respecto a sus propiedades miológicas, difere de Nemertes, Paramphiporus, Correanemertes y Poseidonemertes, usando diagnosis enmendadas por Kirsteuer (1974) en la presencia de musculatura diagonal en la pared del cuerpo. Además se distinguen de Paramphiporus y Poseidonemertes en la ausencia de un septo precerebral; de Nemertes, Paramphiporus y Correanemertes en la posición de los retractores de la cabeza, los cuales en nuestro especímen están relacionados sólo con la porción interior de la musculatura longitudinal. Confrontar con el género Paranemertes es díficil, ya que, no sólo respecto a la doble capa de musculatura longitudinal hay variación inter e intraespecífica, sino que también referente a otros rasgos anatómicos que se citan como genéricos en la diagnosis de Coe (1901) y Friedrich (1955), como son largo del rincoceloma y estructura del ciego intestinal. Kirsteuer (1974) hace un exhaustivo análisis de Paranemertes y dice que: "The species contained in Paranemertes ap- 
parently differ considerably in morphological features, but as long as a definition of the genus can not be based on data verified in the type species it seems futile to speculate which of the taxa should be assigned to other genera"

El grupo hastatus de Friedrich (1955) incluyó las especies: A. hastatus Mc Intosh, 1874 (sensu Oudemans, 1885), A. bioculatus Mc Intosh, 1874 (sensu Joubin, 1890; Bergendal, 1903), A. nebulosus Coe, 1901 y A. korschelti Friedrich, 1939. Estas especies fueron separadas del género Amphiporus por tener en común doble capa de musculatura longitudinal anterior $\mathrm{y}$ un sistema vascular discordante respecto al resto de los Monostilifera. Kirsteuer (1967) considera que A. bioculatus Mc Intosh, 1874 no es igual a A. bioculatus sensu Corrêa (1958) y funda con esta última especie el género Correanemertes. Nuestros especímenes se distinguen de $\mathbf{A}$. hastatus, $\mathbf{A}$. nebulosus y $\mathbf{A}$. korschelti en la organización del sistema vascular; A. hastatus tiene laguna cefálica, A. nebulosus y A. korschelti vasos laterales extracerebrales: Con A. bioculatus Mc Intosh, 1834 es ardua una comparación por la información incompleta disponible acerca de esta especie.

Fasciculonemertes arenicola se separa además de Correanemertes, Poseidonemertes, Paramphiporus, Paranemertes, Nemertes y grupo de especies A. hastatus, por tener células neurocordales en el ganglio cerebroide ventral y canal cerebral bifurcado. Difiere también de Nemertes y de la mayoría de las especies de Paranemertes en el largo del rincoceloma, ya que, en nuestro caso llega hasta el tercio posterior del cuerpo.

La combinación de características presentadas por esta nueva especie no corresponde a los géneros de Monostilifera conocidos, por lo tanto se propone un nuevo género para ella.

\section{AGRADECIMENTOS}

Deseamos agradecer a la Dra. Diva Diniz Corrêa por la revisión crítica del trabajo y sus valiosas sugerencias; al Dr. Patricio Sánchez por su asistencia taxonómica; al Sr. Juan Correa por su asistencia técnica.

\section{LISTA DE ABREVIACONES}

$\begin{aligned} \text { ar } & \text { abertura del rincodeo } \\ \text { cd } & : \text { comisura ganglio dorsal } \\ \text { ci } & : \text { ciego intestinal } \\ \text { cn } & \text { célula neurocordal } \\ \text { d } & \text { dermis } \\ \text { e } & \text { esófago } \\ \text { ep } & \text { epidermis } \\ \text { er } & \text { esfinter del rincodeo } \\ \text { est } & \text { estómago } \\ \text { gc } & \text { glándulas cefálicas } \\ \text { gd } & \text { ganglio dorsal } \\ \text { in } & \text { intestino } \\ \text { ip } & \text { inserción de la proboscis }\end{aligned}$




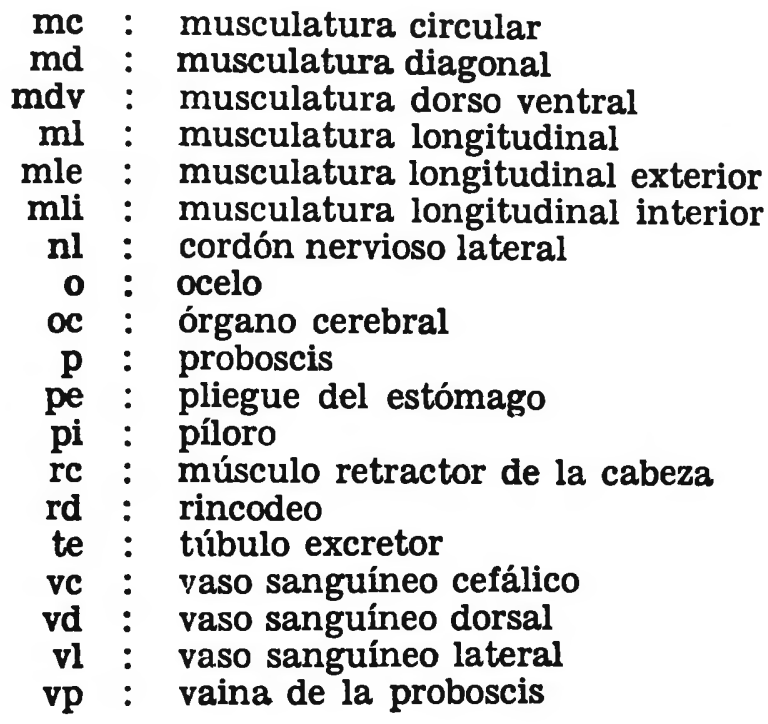

\section{REFERENCIAS}

BERG, G., (1972) - Studies on Nipponnemertes Friedrich, 1968 (Nemertini, Hoplonemertini) I. Redescription of Nipponnemertes pulcher (Johnstcn, 1837) with special reference to intraspecific variation of the taxonomical characteres Zool. Scr. 1: 211-225.

BERGENDAL, D., (1903) - Till kannedomen om de nordiska Nemertinerna. 4. Förteckning ofver Vid Sveriges vestkust iakttagna Nemertiner. Ark. Zool. 1: 85-156.

COE, W. R., (1901) - Papers from the Harriman Alaska Expedition. XX. The Nemerteans. Proc. Wash. Acad. Sci., 3: 1-84.

(1904) - Nemerteans of the Pacific Coast of North America. Part. II. Harriman Alaska Exped., 11: 113-251.

(1905) - Nemerteans of the west and northwest coasts of America. Bull. Mus. comp. Zool. Harv., 47: 1-319.

(1940) - Revision of the nemertean fauna of the Pacific coast of North Central, and northern South America. Allan Hancock Pacif. Exped., 2: 247-323.

(1944) - A new species of hoplonemertean (Paranemertes biocellatus) from the Gulf of Mexico. J. Wash. Acad. Sci., 34: 407-409.

CORREA D.D., (1958) - Nemertinos do litoral brasileiro (VII). Anais Acad. bras Cienc., 29: 441-455.

(1964) - Nemerteans from California and Oregon. Proc. Calif. Acad. Sci., (4) $31: 515-558$.

FRIEDRICH, H., (1939) - Einige neue Hoplonemertinen aus der Ostsee. Kieler Meeresforsch, 3: 233-251.

(1955) - Beitrage zu einer Synopsis der Gattungen der Nemertini monostilifera nebst Bestimmungsschlussel. Z. wiss. Zool., 158: 133-192.

(1970) - Nemertinen aus Chile. Report N. 47 of the Lund University Chile Expedition 1948 - 1949. Sarsia, 40: 1-80.

IWATA, F., (1952) - Nemertini from the Coasts of Kyusyu. J. Fac. Sci. Hokkaido Univ., (6) 11 : 126-148.

(1957) - Nemerteans from Sagami Bay. Publs Akkeshi mar. biol. Stn, 7: $1-31$.

PEDERSEN, K. J., (1968) - Some morphological and histochemical aspects of nemertean connective tissue. Z. Zellforsch mikrosk. Anat. 90: 570-595.

YAMAOKA, T., (1940) - The fauna of Akkeshi Bay. IX. Nemertini. J. Fac. Sci. Hokkaido Univ., (6) 7: 205-263. 